\title{
Supplementary material - Analysis of variability in divergence and turn-over induced by three idealized convective systems with a 3D cloud resolving model
}

Edward Groot ${ }^{1}$ and Holger Tost ${ }^{1}$

${ }^{1}$ Institut für Physik der Atmosphäre, Johannes Gutenberg Universität, Johannes-Joachim-Becher-Weg 21, Mainz, Germany Correspondence: Edward Groot (egroot@uni-mainz.de)

\section{S1 Experiment overview}

Table S1 gives an overview with definitions of all experiments performed in this study. 
Table S1. List of experiments with their respective settings. Each experiment was done with each of the cases.

\begin{tabular}{|c|c|c|}
\hline Name & Experiment collection & Definition \\
\hline Control_reference & All experiment collections & $\begin{array}{l}z_{\text {top }}=6000.0 \mathrm{~m}(\text { case } 1) \\
z_{\text {top }}=2500.0 \mathrm{~m}(\text { case } 2 \& 3) \\
200 \times 200 \times 100 \mathrm{~m} \text { grid } \\
L_{v}=L_{v, r e f} \\
a d v(z)=w \frac{\partial v_{h o r}}{\partial z}, a d v(z)=w \frac{\partial q_{v}}{\partial z}\end{array}$ \\
\hline ENS_01 & Ensemble member & $\begin{array}{l}z_{\text {top }}=6095.9565472 \mathrm{~m} \\
z_{\text {top }}=2539.98189467 \mathrm{~m}\end{array}$ \\
\hline ENS_02 & Ensemble member & $5758.42068902 \mathrm{~m} / / 2399.34195376 \mathrm{~m}$ \\
\hline ENS_03 & Ensemble member & $5887.00610239 \mathrm{~m} / / 2452.91920933 \mathrm{~m}$ \\
\hline ENS_04 & Ensemble member & $6052.55517416 \mathrm{~m} / / 2521.89798923 \mathrm{~m}$ \\
\hline ENS_05 & Ensemble member & $5695.83407152 \mathrm{~m} / / 2373.26419647 \mathrm{~m}$ \\
\hline ENS_06 & Ensemble member & $5744.91637676 \mathrm{~m} / / 2393.71515698 \mathrm{~m}$ \\
\hline ENS_07 & Ensemble member & $5737.47939255 \mathrm{~m} / / 2390.61641356 \mathrm{~m}$ \\
\hline ENS_08 & Ensemble member & $5968.36439833 \mathrm{~m} / / 2486.8184993 \mathrm{~m}$ \\
\hline ENS_09 & Ensemble member & $6095.57941954 \mathrm{~m} / / 2539.82475814 \mathrm{~m}$ \\
\hline ref_res_500m & Resolution (coarse grid) & $500 \times 500 \times 250 \mathrm{~m}$ grid \\
\hline ref_res_1km & Resolution (coarse grid) & $1000 \times 1000 \times 500 \mathrm{~m}$ grid \\
\hline cubic_res_200m & Resolution (fine cubic grid) & $200 \times 200 \times 200 \mathrm{~m}$ grid \\
\hline cubic_res_100m & Resolution (fine cubic grid) & $100 \times 100 \times 100 \mathrm{~m}$ grid \\
\hline controlling_lve_0.6 & Latent heat & $L_{v}=0.6 L_{v, r e f}$ \\
\hline controlling_lve_0.8 & Latent heat & $L_{v}=0.8 L_{v, r e f}$ \\
\hline controlling_lve_0.9 & Latent heat & $L_{v}=0.9 L_{v, r e f}$ \\
\hline controlling_lve_1.1 & Latent heat & $L_{v}=1.1 L_{v, r e f}$ \\
\hline controlling_lve_1.2 & Latent heat & $L_{v}=1.2 L_{v, r e f}$ \\
\hline controlling_vadv_0.0 & Vertical advection of hor. momentum & $a d v(z)=0 \frac{\partial \boldsymbol{v}_{h o r}}{\partial z}$ \\
\hline controlling_vadv_0.5 & Vertical advection of hor. momentum & $a d v(z)=0.5 w \frac{\partial \boldsymbol{v}_{h o r}}{\partial z}$ \\
\hline controlling_vadv_0.8 & Vertical advection of hor. momentum & $a d v(z)=0.8 w \frac{\partial \boldsymbol{v}_{h o r}}{\partial z}$ \\
\hline controlling_vadv_1.5 & Vertical advection of hor. momentum & $a d v(z)=1.5 w \frac{\partial \boldsymbol{v}_{h o r}}{\partial z}$ \\
\hline controlling_qvadv_0.8 & Vertical advection of water vapor & $a d v(z)=0.8 w \frac{\partial q_{v}}{\partial z}$ \\
\hline controlling_qvadv_1.2 & Vertical advection of water vapor & $a d v(z)=1.2 w \frac{\partial q_{v}}{\partial z}$ \\
\hline
\end{tabular}


In Figure S2 the decorrelation between ensemble pairs is shown per case (analogously to and based on Hohenegger and

5 Schär (2007)). The calculation of a zonal velocity deviation field is given in Equation S2. Subsequently, members have been paired to calculate the correlation coefficient between their zonal velocity deviation fields. Because for some simulations initial conditions or final conditions (after 0 and 120 minutes) were not stored properly in the netCDF files, some member pairs of the 10 member ensemble have been omitted. Additionally, if an odd number of members could be used due to such an error, one member has been used in two pairs.

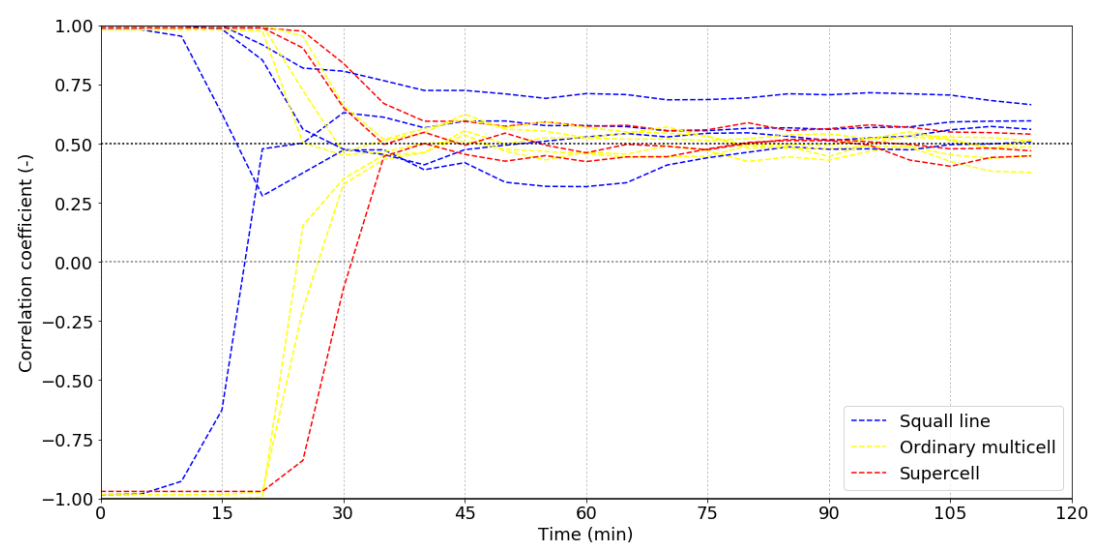

Figure S2. Correlations between zonal velocity deviation fields as function of time. Initially, the errors behave nearly linearly (0-10 minutes), starting from nearly \pm 1 . Then there is a transition stage (10-35 minutes). After the transition stage, all pairs of zonal velocity deviation fields but two (both from the squall line case) seem to behave randomly. After 70 minutes only one has not yet approached the random realization asymptote. 


\section{References}

Hohenegger, C. and Schär, C.: Predictability and Error Growth Dynamics in Cloud-Resolving Models, Journal of the atmospheric sciences, 64, 4467-4478, 2007. 